\title{
Side Lobe Reduction of Concentric Thinned Antenna Array Using Evolutionary Algorithms
}

\author{
D. Mandal ${ }^{1}$, V.P.Roy ${ }^{2}$, A. Chattrejee ${ }^{3}$ A. K. Bhattacharjee ${ }^{4}$ \\ ${ }^{1,2}$ Department of Electronics and Communication Engineering Bengal College of Engineering and Technology, \\ Durgapur, West Bengal, India, \\ ${ }^{3}$ Department of Electronics and Communication Engineering National Institute of Technology, Goa, Goa, India \\ ${ }^{4}$ Department of Electronics and Communication Engineering National Institute of Technology, Durgapur, \\ Durgapur, India \\ Email: deb.mandal22@gmail.com
}

\begin{abstract}
This paper presents how to thin an concentric circular ring array antenna optimally using evolutionary algorithms. In order to obtain lower Sidelobe level (SLL) form a large antenna array in different azimuth planes, various types of optimization algorithms have been used. A pattern synthesis method based on thinning is presented to generate a pencil beam pattern from a concentric ring array of isotropic antennas. It is obtained by finding out optimum sets of elements state using GA, PSO and DE in four azimuth planes . Results clearly show the effectiveness of the proposed method.
\end{abstract}

Keywords: concentric circular ring array, genetic algorithm (GA), differential evolution Algorithm $(D E)$, particle swarm optimization Algorithm (PSO), peak sidelobe level (peak SLL), thinned array

\section{Introduction}

Due to 360 degree azimuthally symmetrical beam pattern with compact structure Concentric Circular Antenna Array (CCAA) plays an important role in wireless and radar communication [1,2]. Uniformly excited and equally spaced antenna arrays have high directivity, but it suffers from high SLL. To reduce the high SLL, the array thinned using evolutionary algorithms. Several approaches reported in the literature for generating radiation pattern from thinned antenna array [1-7] are as follows:

R.L.Haput proposed several ways to apply genetic algorithm to optimized array pattern [3]. QuevedoTeruel et al. proven the usefulness of ACO to design arrays by thinned methods using the SLL as optimization parameter [4]. Mahanti et al. proposed a technique for designing a thinned linear antenna array with fixed sidelobe level and fixed percentage of thinning using global optimization tool [5]. A pattern synthesis method based on thinning using Boolean Differential Evolution Algorithm and FFT for planner array has been reported by Zhang, L.[6]. R. Jain et al. discussed the use of genetic algorithm for thinned linear and planar antenna arrays and also pointed out the problems of applying simple GA to a larger array under dynamic conditions [7]. However, due to complexity of synthesis problem it cannot be solved with analytical methods. Therefore, evolutionary optimization methods are a good option to solve these problems. Among them, genetic algorithms (GA)[7-11], particle swarm optimization (PSO)[12-15], and differential evolution algorithm (DE)[6, 16-18] have already been used in array synthesis for different requirements but most of the literature produces an array pattern using thinning in a single azimuth plane. In this paper, author proposed a comparative study based on three different optimization algorithms for thinning large multiple concentric circular ring arrays of isotropic antennas that reduced the side lobe level in a number of different azimuth planes.

\section{Problem Formulation}

A concentric array of isotropic elements is considered. The far field pattern of the array shown in Figure 1. can be written as $[1-2,14$, and 18]:

$A F(\theta, \varphi)=\sum_{m=1}^{M} \sum_{n=1}^{N_{m}} I_{m n} e^{j\left[k r_{m} \sin \theta \cos \left(\varphi-\varphi_{m n}\right)+\alpha_{m n}\right]}$

where, $M=$ number of concentric rings; $N_{m}=$ number of isotropic elements in $m$-th ring; $I_{m n}=$ excitation amplitude of $m n$-th element; $I_{m n}=1$ if $m n$-th element is turned "on" and 0 if it is "off". The $r_{m}=N_{m} d_{m} / 2 \pi$ radius of the $m$-th ring; $d_{m}=$ inter element arc spacing of $m$-th circle; $k=2 \pi / \lambda$, represents wave number; $\lambda$ is the wave length; $\theta, \varphi=$ polar and azimuth angle; $\varphi_{m n}=2 n \pi / N_{m}$ is the angular location of the $m n$-th element with, $1 \leq n \leq N_{m}$ and $\alpha_{m n}=$ phase excitation of $m n$-th element consider as zero degree for each. 


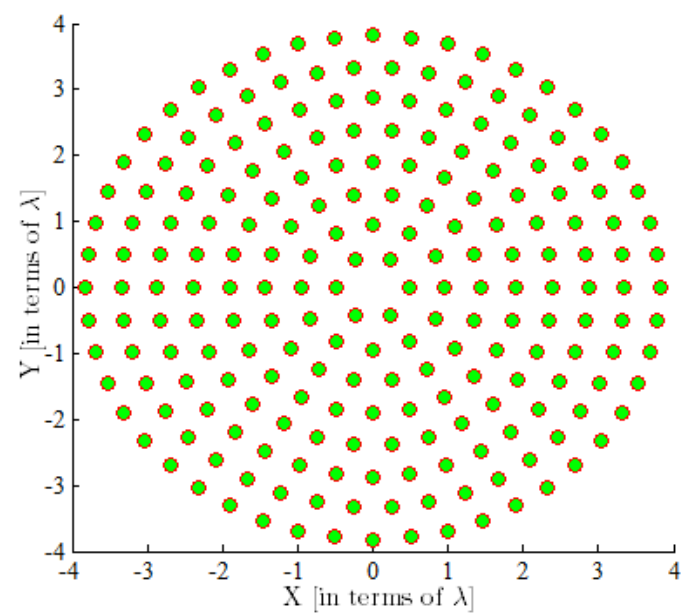

Figure 1: Concentric ring array of isotropic antennas in $\mathrm{X}-\mathrm{Y}$ plane.

Normalized power pattern $P(\theta, \varphi)$ in $\mathrm{dB}$ can be expressed as follows:

$P(\theta, \varphi)=10 \log 10\left[\frac{\mid A F(\theta, \varphi)}{|A F(\theta, \varphi)|_{\max }}\right]^{2}=20 \log 10\left[\frac{\mid A F(\theta, \varphi)}{|A F(\theta, \varphi)|_{\max }}\right]$

The directivity is a parameter which quantifies the radiation focusing capability of an antenna.If we define the normalized radiation pattern $A F_{n}(\theta, \varphi)=\left[\frac{A F(\theta, \varphi)}{A F(\theta, \varphi)_{\max }}\right]$

The directivity can be written as:

$$
D=\left[\frac{4 \pi}{\int_{\theta=0}^{\pi} \int_{\varphi=0}^{2 \pi}\left|A F_{n}(\theta, \varphi)\right|^{2} \sin \theta d \theta d \varphi}\right]
$$

The fitness function for the beam pattern is defined as:

$$
F(\rho)=k_{1}\left[\text { peakSLL } L^{d}-\max _{\theta \in A}\left\{A F_{d B}^{\rho}(\theta, \varphi)\right\}\right]^{2} H(X)
$$

In equation (4) $\varphi \in\left(0^{\circ}-90^{\circ}\right)$ and $\rho$ is the unknown parameter set responsible for the beam patterns. $\rho$ is defined as follows: $\rho=\left\{I_{m n}\right\} ; \quad\left\{\begin{array}{c}1 \leq m \leq M \\ 1 \leq n \leq N_{m}\end{array}\right.$

$H(X)$ is Heaviside step function defined as follows:

$$
X=\left[\operatorname{peakSLL}^{d}-\max _{\theta \in A}\left\{A F_{d B}^{\rho}(\theta, \varphi)\right\}\right]
$$

$H(X)=\left\{\begin{array}{lll}1 & \text { if } & X \geq 0 \\ 0, & \text { if } & X<0\end{array}\right.$

peakSLL ${ }^{d}$ is the desired value of peak SLL. $A$ is the sidelobe region. $\max _{\theta \in A}\left\{A F_{d B}^{\rho}(\theta, \varphi)\right\}$ represents the obtained maximum side-lobe level of the beam-pattern within the pre-specified range of $\varphi$-plane for the unknown parameter set $\rho$, which has to be determined. The term, $\left[\operatorname{peakSLL} L^{d}-\max _{\theta \in A}\left\{A F_{d B}^{\rho}(\theta, \varphi)\right\}\right]$ represent the deviation between the desired and obtained values of the peak SLL for the parameter set $\rho$. 
Where $\varphi=0^{\circ}, 30^{\circ}, 60^{\circ}, 90^{\circ}$ plane and $k_{1}$ is the weighting factors. For optimal synthesis of pattern, the fitness function of equation (3) has to be minimized using GA, PSO and DE.

Number of elements does not contribute to construct beam pattern after thinning in comparison to a fully populated array can be expressed in terms of tapper efficiency [7]. An array taper efficiency can be calculated from

$$
\eta_{a r}=\left(\frac{\text { number of elementsin the array turned off }}{\text { total number of elements in the array }} \times 100\right) \%
$$

\section{Overview of Differential Evolution Algorithm}

\section{Algorithm Overviews And Parametric Setup}

Differential Evolution Algorithm (DE) was introduced by Storn and Price. Similar to GA [7-11], DE is also based on population. It is a stochastic optimization method used to minimize an objective function. It has an advantage to find out a true global minima and fast convergence using a few control parameters [16-18]. DE algorithm generates a population of $N P$ in $D$ dimensional search space called individuals. The individual of population in generation $G$ can be written as:

$$
X_{i, G}=\left\{x_{1 i, G}, x_{\left.2 i, G \cdots \cdots . . x_{D i, G}\right\}, i=1,2 \ldots \ldots \ldots . N P .}\right.
$$

The initial population covered the entire search space. At a generation $G=0$ the initial value of the jth parameter defined as follows:

$$
x_{j i, 0}=\operatorname{rand}(0,1) \cdot\left(x_{j}^{u p}-x_{j}^{l o w}\right)+x_{j}^{l o w}
$$

where $i=1,2, \ldots \ldots \ldots . . N P$ and $j=1,2, \ldots \ldots \ldots . . D$. $\operatorname{rand}(0,1)$ is uniformly distributed random variable within the

range $(0,1) ; x_{j}^{\text {low }}$ and $x_{j}^{u p}$ are lower and upper bounds of $j-$ th parameter. Three steps mutation, crossover and selection can be described as follows.

Mutation operation: DE performs mutation operation to generate a mutant vector $V_{i, G}=\left\{v_{1 i, G}, v_{2 i, G}, \ldots, v_{D i, G}\right\}$

for each target vector $X_{i, G}$. In this work, the DE strategy used is: ' $D E /$ best $/ 1 /$ bin' which is defined as follows [6, 16-18]:

$V_{i, G}=X_{\text {best }, G}+F \cdot\left(X_{r 1, G}-X_{r 2, G}\right)$

$r_{1}, r_{2} \in[1, N P]$ and $r_{1} \neq r_{2} \neq i . F$ is a real and constant factor, satisfies $F \in[0,2]$ and $X_{b e s t, G}$ is the vector which has best fitness at $G^{\text {th }}$ generation.

Crossover operation: In this operation trial vector

$$
U_{i, G}=\left\{u_{1 i, G}, u_{2 i, G}, \ldots, u_{D i, G}\right\}
$$

is generated from the target vector $X_{i, G}$ and mutant vector $V_{i, G}$. The crossover strategy is defined follows:

$$
u_{j i, G}=\left\{\begin{array}{l}
v_{j i, G} \text { if rand }(0,1) \leq C R \\
x_{j i, G} \text { otherwise }
\end{array}\right.
$$

Crossover factor $C R$ is constant in the range of $(1,0)$. The value of $C R$ is taken as: 0.2 .

Selection: The operation performs comparison between the objective function values at each trial vector $f\left(U_{i, G}\right)$ and target vector $f\left(X_{i, G}\right)$. The vector which has smaller fitness function value remains in the next generation. Selection operation can be expressed as

$$
X_{i, G+1}=\left\{\begin{array}{l}
U_{i, G} \quad \text { if } f\left(U_{i, G}\right)<f\left(X_{i, G}\right) \\
X_{i, G} \text { otherwise }
\end{array}\right.
$$




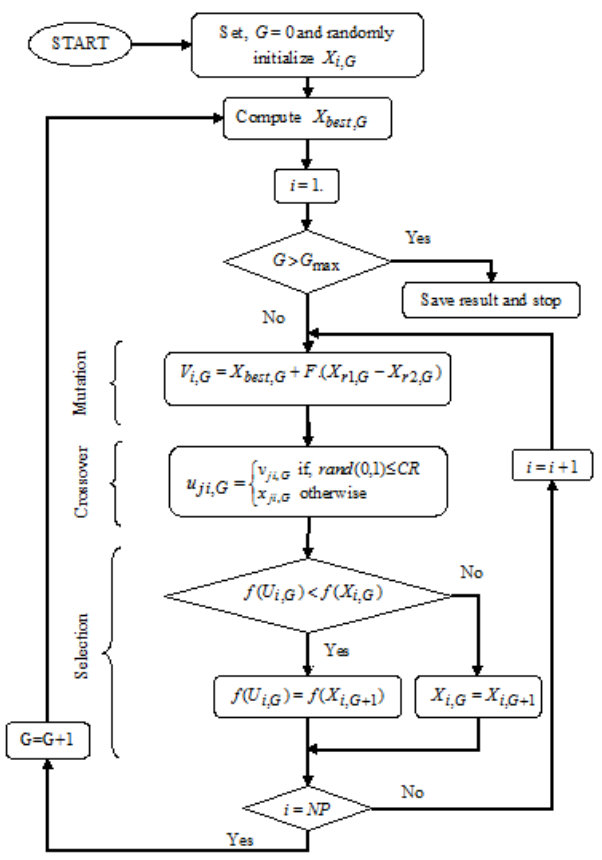

Figure 2. Flow chart of Differential Evolution (DE) algorithm.

These three steps are repeated generation by generation until it reaches to its termination condition. Return the best vector in the current population $\left(X_{\text {best }, G}\right)$ as the solution of the optimization problem. The flow chart of Differential Evolution is given in Fig 2.

\section{Details OF PARAMETRIC SETUP}

The individual of the population for DE, PSO and GA are considered as:

$\rho=\left[\begin{array}{llll}I_{1} & I_{2} & \ldots & I_{K}\end{array}\right]$

Since the quantity $I_{m}$ has only two state i.e. ' $o n$ ' and 'off'. The limits of the variable are defined as:

$I_{m}=\left\{\begin{array}{llll}1 & \text { ('on') } & \text { if }, & 0.5 \leq I_{m} \leq 1 \\ 0 & \text { ('off') } & \text { if }, & 0 \leq I_{m}<0.5\end{array} \quad\right.$ for $m=1,2, \ldots, K$

Here 'on' represent the subset of element number which are ON. The value of $K$ in this problem becomes 216 and the search space dimension becomes 216 .

Based on the guideline provided in [6,16-18], the population size, and scale factor $(F)$, and crossover rate $(C R)$ of DE are chosen as: $50,0.8$ and 0.2 . The $\mathrm{DE}$ scheme used is: 'DE/best/1/bin' and the maximum iteration number is chosen as: 3000 .

Swarm size in PSO is taken as 50 and the initial population is chosen randomly. The value of $C 1$ and $C 2$ are chosen as 2 [12-15]. Time-varying inertia weight $(w)$ is considered as decreases linearly from 0.9 to 0.4 . The maximum allowable velocity for each of the particle on $d$-th dimension is considered as $0.9 r_{d}$ [12-15], where $r_{d}$ is the difference between the maximum and minimum possible values of decision variables on $d$-th dimension. The termination condition is chosen as a maximum iteration of 3000 .

Population size in GA is taken as 50 and two-point crossover is chosen. Crossover probability and mutation probability are taken as: 0.08 and 0.01 and 'Roulette Wheel' Selection is considered for the proposed problem and the termination condition is chosen as a maximum iteration of 3000. Other parametric setups of GA are taken from guidelines given in [7-11].

\section{Simulation Results}

A circular array of eight concentric rings has been considered and each rings contained a number of isotropic elements separated by a distance $0.5 \lambda$ having ring radius $r_{m}(\lambda)$ shown in Table 1.The design specification of the array pattern and its corresponding obtained results are shown in Table 2. The peak SLL for the array pattern using different Evolutionary Algorithms corresponding to its predefined azimuth plane $\varphi=0^{\circ}, 30^{\circ}, 60^{\circ}$ and $90^{\circ}$ mentioned in Table 2 . along with its desired values. The optimized array and obtained 
array patterns are shown in Fig.3 and Fig.4. Thinned array using different Optimization Algorithms are shown in Fig.3. In Fig.3(a). the array is thinned using Genetic Algorithm, in Fig.3(b). using Particle Swarm Optimization

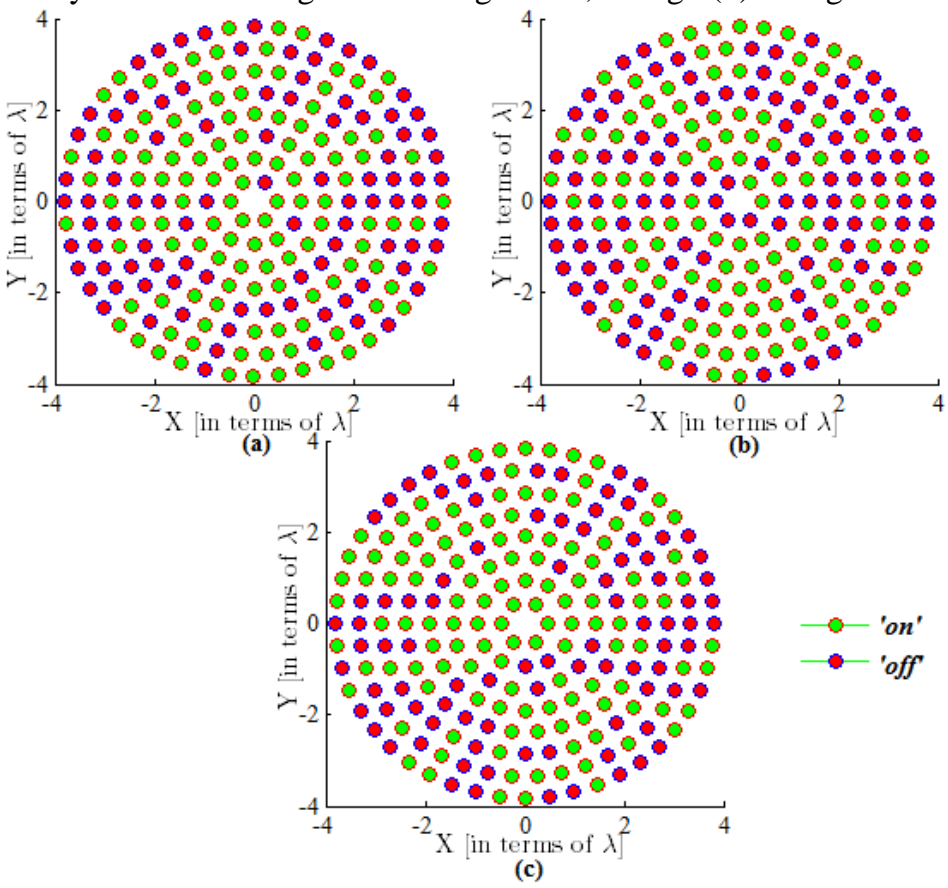

Figure 3: Optimized Concentric Ring array using (a) GA (b) PSO (c) DE.

Algorithm and Fig.3(c). using Differential Evolution Algorithm. The array factor in dB against the Elevation angle for predefined Azimuth planes using different Optimization Algorithms is plotted in Fig.4. Table 2. depicts the maximum values of peak SLL along with the corresponding directivity for different Optimization Algorithms in four various Azimuth planes. The best set of obtained values of PeakSLL and directivity are achieved using DE in every azimuth planes. The state of the elements is given in Table 3. The element is considered as "on"/ "off" according to its corresponding state " 1 "/ " 0 " shown in Table 3 . Here when the element is fed is said to be "on" and "off" when the element is passively terminated by impedance equal to the source impedance of the fed elements. Best Fitness values are plotted against Generations or number of iterations to get the convergence characteristic shown in Fig.5. The effectiveness of DE over GA and PSO are clearly shown in Fig.5. All computations have been done in MATLAB 2010a on core 2 duo processor, 3GHz with 2 GB RAM.

Table 1: Ring radius and number of elements per ring

\begin{tabular}{|c|c|c|c|c|c|c|c|c|}
\hline $\mathrm{M}$ & 1 & 2 & 3 & 4 & 5 & 6 & 7 & 8 \\
\hline$r_{m}(\lambda)$ & $\underset{\stackrel{n}{f}}{\stackrel{\mathfrak{f}}{+}}$ & $\begin{array}{l}\text { 高 } \\
\text { ó }\end{array}$ & $\stackrel{\stackrel{J}{J}}{\stackrel{\sim}{\sim}}$ & $\begin{array}{l}\text { ळे } \\
\text { }\end{array}$ & $\begin{array}{l}\stackrel{n}{\infty} \\
\stackrel{\infty}{i} \\
\stackrel{n}{i}\end{array}$ & \begin{tabular}{l}
$\infty$ \\
\multirow{d}{0}{} \\
$\infty$ \\
$i$
\end{tabular} & $\underset{\tilde{I}}{\tilde{m}}$ & $\begin{array}{l}\hat{\sigma} \\
\dot{\infty} \\
\dot{n}\end{array}$ \\
\hline$N_{m}$ & 6 & 12 & 18 & 24 & 30 & 36 & 42 & 48 \\
\hline
\end{tabular}

Table 2: Obtained Results for Different Algorithms In Different $\varphi$ Planes

\begin{tabular}{|c|c|c|c|c|}
\hline Optimization & \multirow{2}{*}{$\varphi$ in degrees } & \multicolumn{2}{|c|}{ PealSLL in dB } & \multirow{2}{*}{ Directivity } \\
\cline { 2 - 5 } Algorithms & & Desired & Obtained & \\
\hline & 0 & -15.00 & -26.78 & \multirow{2}{*}{24.6424} \\
Genetic Algorithm (GA) & 30 & -15.00 & -26.82 & \\
& 60 & -15.00 & -26.98 & \\
& 90 & -15.00 & -26.96 & 24.3636 \\
Particle Swarm & 0 & -15.00 & -22.80 & \\
Optimization (PSO) & 30 & -15.00 & -22.19 & \\
& 60 & -15.00 & -22.09 & 24.9969 \\
Differential Evolution & 90 & -15.00 & -22.06 & \\
Algorithm (DE) & 0 & -15.00 & -27.37 & -27.36 \\
\end{tabular}



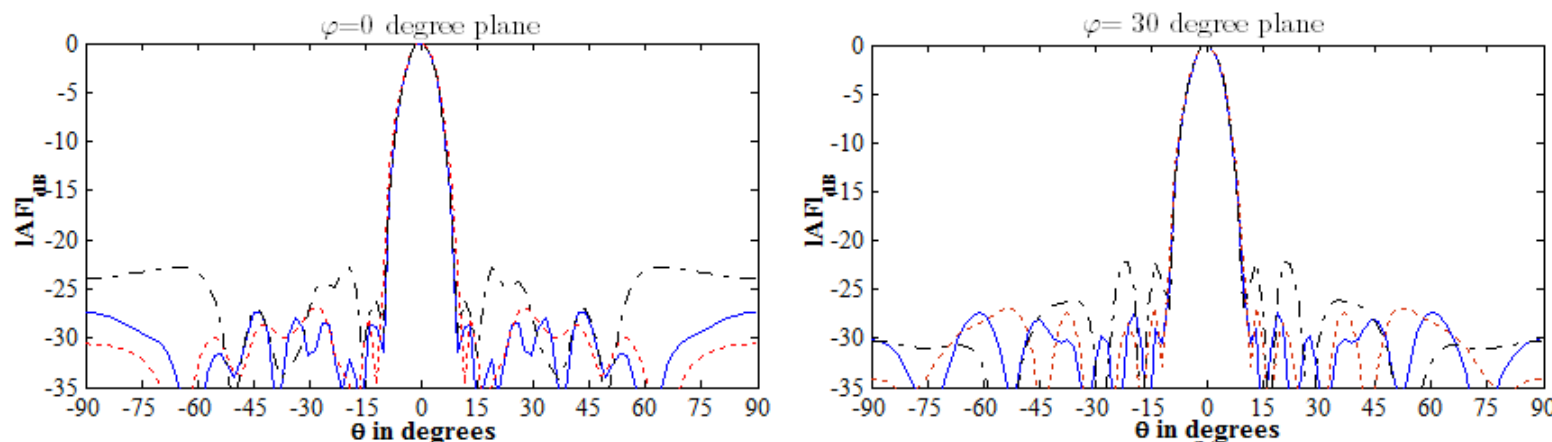

(a)

(b)

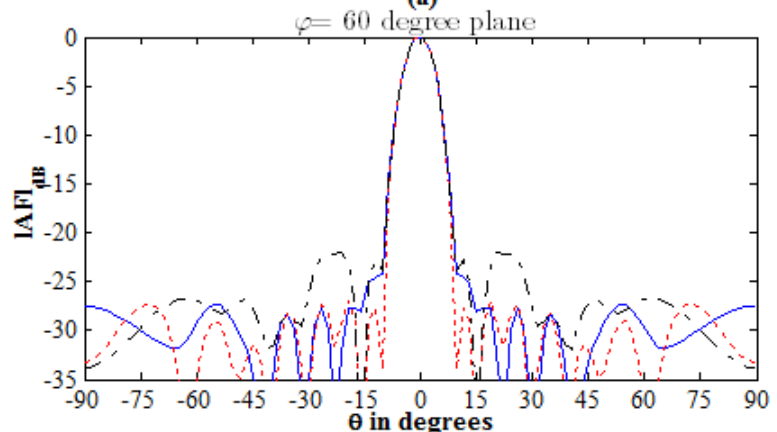

(c)

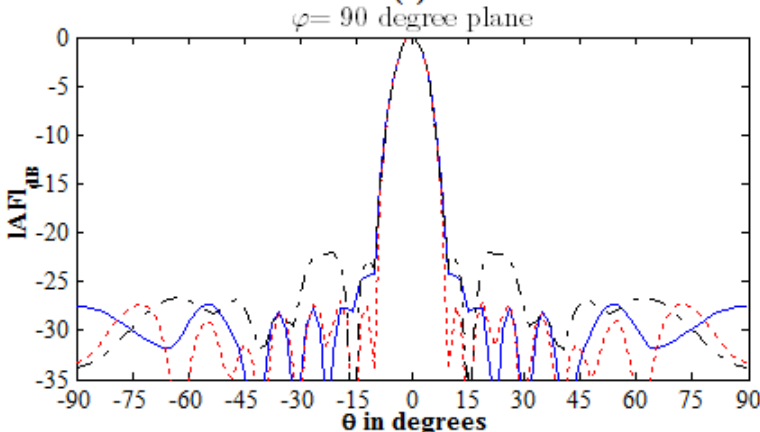

(d)

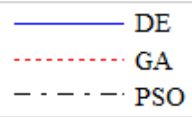

Figure 4: Array Pattern investigated using Evolutionary Algorithms: for (a) $\varphi=0^{\circ}$ (b) $\varphi=30^{\circ}$

$$
\text { (c) } \varphi=60^{\circ} \text { (d) } \varphi=90^{\circ} \text {. }
$$

Table 3. State of the element in each ring

\begin{tabular}{|c|c|c|}
\hline 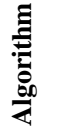 & 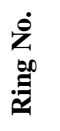 & Elements States Ring wise \\
\hline 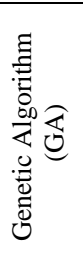 & $\begin{array}{l}1 \\
2 \\
3 \\
4 \\
5 \\
6 \\
7 \\
8\end{array}$ & $\begin{array}{l}1,0,1,1,1,1 \\
1,1,1,1,1,1,0,1,1,1,1,0 \\
1,0,1,1,0,1,1,1,1,1,0,1,1,1,1,1,1,1 \\
0,1,1,1,1,1,1,1,0,1,1,1,0,1,1,0,0,1,1,1,0,0,0,0 \\
0,0,1,1,0,1,0,0,1,1,1,1,0,1,1,0,1,0,0,0,1,1,0,0,0,1,1,1,1,1 \\
0,0,0,1,0,0,1,0,1,1,1,1,0,0,1,1,1,0,1,0,1,0,0,1,0,1,0,1,1,1,0,0,0,1,0,1 \\
0,0,1,0,0,1,1,1,0,0,1,0,1,1,1,1,0,0,1,0,1,0,0,0,0,0,1,0,1,1,0,1,1,1,0,1,0,1,1,0,0,1 \\
1,0,0,0,0,0,1,0,0,0,1,1,0,1,0,0,0,0,1,1,0,0,1,0,0,1,0,0,0,0,1,1,1,1,0,1,1,1,1,1,1,1,0,1,0,1,0,0\end{array}$ \\
\hline 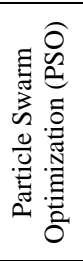 & $\begin{array}{l}1 \\
2 \\
3 \\
4 \\
5 \\
6 \\
7 \\
8\end{array}$ & $\begin{array}{l}1,1,0,0,0,0 \\
0,1,0,1,1,0,1,1,1,1,1,0 \\
0,1,0,1,1,1,1,1,0,0,1,0,0,1,1,1,1,1 \\
0,0,1,0,0,1,1,1,1,0,0,1,0,1,1,0,1,1,1,1,1,0,1,0 \\
1,0,0,0,1,0,0,0,0,0,1,1,0,0,1,1,0,1,1,1,0,0,1,1,1,0,1,1,0,0 \\
1,0,0,1,0,0,0,1,0,1,1,0,1,1,1,1,0,1,0,1,0,0,1,0,0,1,1,1,1,1,0,1,1,0,1,1 \\
0,1,0,0,1,0,0,1,1,0,0,1,1,0,0,1,0,0,1,1,0,1,0,0,0,0,1,0,0,1,1,1,1,1,1,1,1,1,0,1,0 \\
0,0,1,0,0,0,0,1,1,0,1,1,1,1,1,1,0,0,1,0,0,1,0,1,0,0,0,0,0,0,1,0,0,1,0,1,1,0,0,0,0,0,1,1,1,1,1,0\end{array}$ \\
\hline 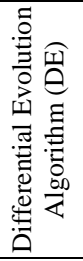 & $\begin{array}{l}1 \\
2 \\
3 \\
4 \\
5 \\
6 \\
7 \\
8\end{array}$ & $\begin{array}{l}1,1,1,1,1,1 \\
1,1,1,1,1,1,1,1,1,0,0,1 \\
1,1,1,0,1,1,1,1,1,1,1,1,0,1,0,1,0,0 \\
1,0,0,1,1,1,1,1,0,1,0,0,1,1,1,0,1,1,1,1,1,1,0,1 \\
0,1,1,0,1,0,0,0,1,1,1,1,1,1,0,1,0,1,1,0,0,0,1,1,1,1,1,1,0,1 \\
0,1,0,0,0,1,0,1,1,1,1,0,1,1,1,1,1,0,1,0,1,0,0,0,1,0,1,0,0,1,1,0,1,0,0,0 \\
0,0,1,1,0,1,0,0,1,0,0,1,0,0,0,1,1,1,1,1,0,0,0,1,0,0,1,0,1,0,0,1,1,1,1,0,1,0,1,0,1,0 \\
0,0,0,0,0,1,1,0,0,1,1,1,1,1,1,1,0,0,0,0,1,1,1,1,0,1,0,1,0,0,0,1,1,0,0,1,1,0,1,1,0,0,0,1,1,0,1,1\end{array}$ \\
\hline
\end{tabular}




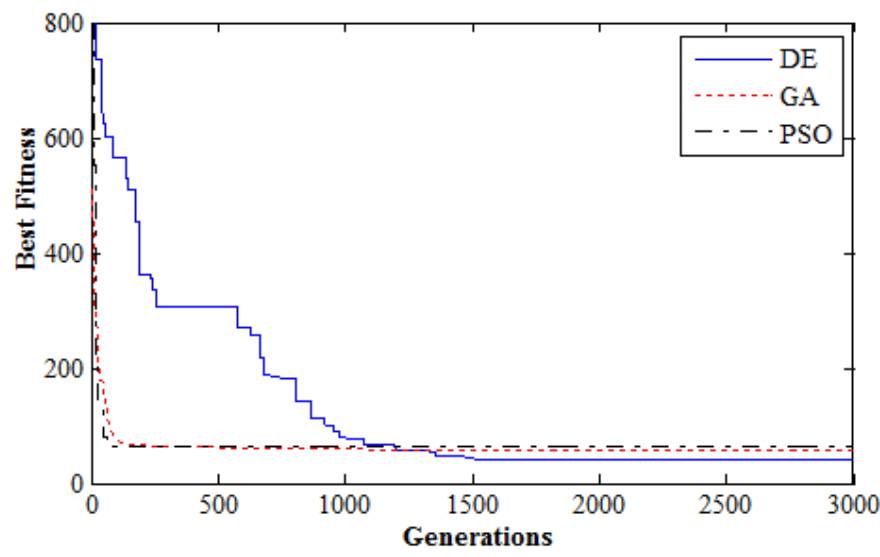

Figure 5: Convergence curve for DE, GA, PSO

\section{Conclusion}

The radiation pattern from a concentric ring array of isotropic elements using Evolutionary Algorithms is presented. By finding out optimum states ("on"/"off") of the array elements peakSLL is reduced. The fitness function takes an important role in this problem. The presented problem is a unique from other array pattern synthesis in this manner, it presents one optimum element state to determine beam patterns with lower peak SLL for four different phi-cut of the pattern, the directivity of the beam patterns are also computed. Results clearly indicate the effectiveness of DE over PSO and GA. The presented method can also be applied to synthesize other array configurations.

\section{References}

[1]. C. A. Balanis, Antenna Theory: Analysis and Design, 2nd ed. Wiley-IEEE Press, 1997.

[2]. R. Elliott, Antenna Theory and Design, revised ed. John Wiley \& Sons Inc, 2003.

[3]. R. L. Haupt, "Thinned arrays using genetic algorithms," Antennas and Propagation IEEE Transactions, vol. 42, no. 7, pp. 993-999, Jul. 1994.

[4]. E. Quevedo-Teruel, O.; Rajo-Iglesias, "Ant Colony Optimization in Thinned Array Synthesis With Minimum Sidelobe Level," Antennas and Wireless Propagation Letters, IEEE, vol. 5, no. 1, pp. 349-352, Dec. 2006.

[5]. G. K. Mahanti, N. N. Pathak, and M. P. K., "Synthesis of thinned linear antenna arrays with fixed sidelobe level using real-coded genetic algorithm," Progress In Electromagnetics Research, vol. 75, pp. 319-328, 2007.

[6]. L. Zhang, Y. C. Jiao, Z. B. Weng, and F.-S. Zhang, "Design of planar thinned arrays using a Boolean di_erential evolution algorithm," Microwaves Antennas and Propagation IET, vol. 4, no. 12, pp. 2172 -2178, Dec. 2010.

[7]. R. Jain and G. S. Mani, "Solving Antenna Array Thinning Problem Using Genetic Algorithm," Applied Computational Intelligence and Soft Computing, vol. 2012, p. 14, 2012.

[8]. R. L. Haupt, "An introduction to genetic algorithms for electromagnetics," Antennas and Propagation Magazine, IEEE, vol. 37, no. 2, pp. 7-15, Apr 1995.

[9]. K. Man, K. Tang, and S. Kwong, "Genetic algorithms: concepts and applications [in engineering design]," Industrial Electronics, IEEE Transactions on, vol. 43, no. 5, pp. 519-534, Oct 1996.

[10]. D. Marcano and F. Duran, "Synthesis of antenna arrays using genetic algorithms," Antennas and Propagation Magazine, IEEE, vol. 42, no. 3, pp. 12-20, Jun 2000 .

[11]. D. Mandal and A. Bhattacharjee, "Synthesis of cosec2 pattern of circular array antenna using genetic algorithm," in Communications, Devices and Intelligent Systems (CODIS), 2012 International Conference on, Dec 2012, pp. 546-548.

[12]. J. Kennedy and R. Eberhart, "Particle swarm optimization," in Neural Networks, 1995. Proceedings., IEEE International Conference on, vol. 4, Nov 1995, pp. 1942-1948 vol.4.

[13]. X.-H. Shi and R. C. Eberhart, "Empirical study of particle swarm optimization," in Proceedings of the Congress on Evolutionary Computation, 1999, pp. 1945-1950.

[14]. A. Chatterjee, G. K. Mahanti, and C. A., "Design of a fully digital controlled reconfigurable switched beam concentric ring array antenna using firefly and particle swarm optimization algorithm," Progress In Electromagnetic Research B, vol. 36, pp. 113-131, 2012.

[15]. D. Mandal, K. Kola, and A. Bhattacharjee, "Sidelobe Reduction of a Scanned Circular Array using Particle Swarm Optimization Algorithm," International Journal of Recent Trends in Electrical and Electronics Engg, vol. 3, no. 1, pp. 54-61, Dec. 2013.

[16]. R. Storn and K. Price, "Differential Evolution - A Simple and Efficient Heuristic for global Optimization over Continuous Spaces," Journal of Global Optimization, vol. 11, pp. 341-359, 1997.

[17]. K. V. Price, R. M. Storn, and J. A. Lampinen, "Differential Evolution-A Practical Approach to Global Optimization. Natural Computing," Springer-Verlag, 2005

[18]. D. Mandal, A. Chatterjee, and A. K. Bhattacharjee, "Design of Fully Digital Controlled Shaped Beam Synthesis Using Differential Evolution Algorithm,” International Journal of Antennas and Propagation, vol. 2013, pp. 1-9, 2013. 